\title{
A Simple Estimation of Coupling Loss Factors for Two Flexible Subsystems Connected via Discrete Interfaces
}

\author{
Jun Zhang, ${ }^{1,2}$ Lin Ji, ${ }^{3}$ Zhenyu Huang, ${ }^{4}$ Pingping Zhang, ${ }^{3}$ and Wei Wang ${ }^{4}$ \\ ${ }^{1}$ Changan Auto Global R\&D Center, Chongqing, China \\ ${ }^{2}$ State Key Laboratory of Vehicle NVH and Safety Technology, Chongqing, China \\ ${ }^{3}$ School of Mechanical Engineering, Shandong University, Jinan, China \\ ${ }^{4}$ Institute of Intelligent Mechatronics Research, Shanghai Jiao Tong University, Shanghai, China
}

Correspondence should be addressed to Lin Ji; jilin@sdu.edu.cn

Received 15 July 2016; Accepted 30 October 2016

Academic Editor: Carlo Rainieri

Copyright (c) 2016 Jun Zhang et al. This is an open access article distributed under the Creative Commons Attribution License, which permits unrestricted use, distribution, and reproduction in any medium, provided the original work is properly cited.

\begin{abstract}
A simple formula is proposed to estimate the Statistical Energy Analysis (SEA) coupling loss factors (CLFs) for two flexible subsystems connected via discrete interfaces. First, the dynamic interactions between two discretely connected subsystems are described as a set of intermodal coupling stiffness terms. It is then found that if both subsystems are of high modal density and meanwhile the interface points all act independently, the intermodal dynamic couplings become dominated by only those between different subsystem mode sets. If ensemble- and frequency-averaged, the intermodal coupling stiffness terms can simply reduce to a function of the characteristic dynamic properties of each subsystem and the subsystem mass, as well as the number of interface points. The results can thus be accommodated within the theoretical frame of conventional SEA theory to yield a simple CLF formula. Meanwhile, the approach allows the weak coupling region between the two SEA subsystems to be distinguished simply and explicitly. The consistency and difference of the present technique with and from the traditional wave-based SEA solutions are discussed. Finally, numerical examples are given to illustrate the good performance of the present technique.
\end{abstract}

\section{Introduction}

Statistical Energy Analysis (SEA) [1] is commonly used for higher frequency modelling of complex systems. In SEA, the whole structure is firstly partitioned into many individual SEA subsystems with joined interfaces, and then the power balance equation is set up for each subsystem by equating the power input into the subsystem to that of output, including both the power dissipated within the subsystem itself by the subsystem damping and that transmitted to other subsystems via the subsystem interfaces. A key assumption which underpins the set-up of these power balance equations is that the transmitted power between any two SEA subsystems is proportional to the energy difference between them, and the proportionality is determined by the so-called "coupling loss factors (CLFs)" between the two subsystems [1]. Although a rigorous proof is still under investigations $[2,3]$, it has been widely accepted that the coupling power proportional (CPP) assumption is generally valid if all the SEA subsystems are highly modal dense and weakly coupled [4-9]. It is seen therefore that a successful application of the SEA theory somehow largely relies on the following two aspects: (1) a clear distinguishing of the weak coupling region and (2) an accurate estimation of the coupling loss factors between coupled SEA subsystems. These actually have formed the two main issues of the SEA modelling techniques in the past decades and resulted in extensive research efforts from the SEA researchers, such as [10-16]. A good summary of the large quantity of the relevant publications can be found in [17].

Regarding simple estimations of CLFs for discretely connected subsystems, the existing SEA literature shows that simple formulas of CLF estimations are only available for limited coupling cases so far, for example, plate to room/cavity, room to room/cavity, plate to plate with a line connection, and structure to structure with a single-point connection $[1,18]$. For multipoint couplings between SEA subsystems, 
for example, the solar panels in a spacecraft [19], however, there is no such simple formula available. It is true that [1] did propose two simple formulas for CLF estimations between two subsystems connected via a single point by using wave approach (10.1.6) and then by a modal approach (10.1.18). But, as stated in [1] by Lyon and DeJong, the derivations were only rigorous for one-dimensional subsystems and incoherence waves. It thus becomes rather arguable whether the two formulas can be extended straightforward to two- and threedimensional subsystems with multipoint couplings in that more implicit/explicit assumptions have to be involved.

For multipoint couplings, on one hand, one can generally rely on the principle of power injection methods (PIMs) [20] to predict the SEA CLFs, either experimentally [21] or numerically $[22,23]$, despite such a fact that PIMs are generally expensive and also lack of the ability to measure the coupling strengths between SEA subsystems. On the other hand, many specialist methods are also available which were mainly proposed from finite elements using modal bases of uncoupled subsystems, for example [12-15]. These specialist approaches are found to be much more accurate and efficient than PIMs. However, these methods still need a certain level of extra knowledge of the modes of the uncoupled subsystems except for the gross modal properties. It is therefore very desirable if SEA CLFs can be determined only from the gross modal properties of SEA subsystems and the number of interface degrees of freedom, and, preferably, the predicting technique also allows the weak coupling region to be distinguished in a simple and explicit manner.

With this in mind, the paper aims to propose a simple formula to estimate the SEA CLFs for multipoint coupling cases together with a simple measurement of the coupling strength level between SEA subsystems. The derivation procedure is closely in line with the theoretical frame of [1], but the intermodal stiffness coupling terms are employed in the present derivation instead of the wave transmission coefficient terms in [1]. By assuming each subsystem being of high modal density and the interface degrees of freedom act independently from each other, the statistical coupling relations between the two sets of subsystem modes can be put into a very simple form. It thus allows a simple CLF formula to be derived by accommodating with the results given in $[1,2]$. In addition, the predicting procedure allows the weak coupling region between the two subsystems to be determined simply but explicitly. Compared with other forms of CLF solutions, the proposed technique is found to be extremely easy to apply.

The paper is organized as follows. First, a subsystem modal analysis technique, as described in [24], is used to find out the theoretical relations (both analytical and statistical) of the intermodal coupling stiffness between two generic SEA subsystems with discrete interfaces. Then, in Section 3, the statistics of the intermodal coupling stiffness terms are accommodated within the theoretical frame of $[1,2]$ to yield two simple formulas: one for SEA CLF estimation and the other for coupling strength measurement. Finally, numerical examples of two plates connected via discrete points are provided in Section 4 to illustrate the good performance of the proposed technique.

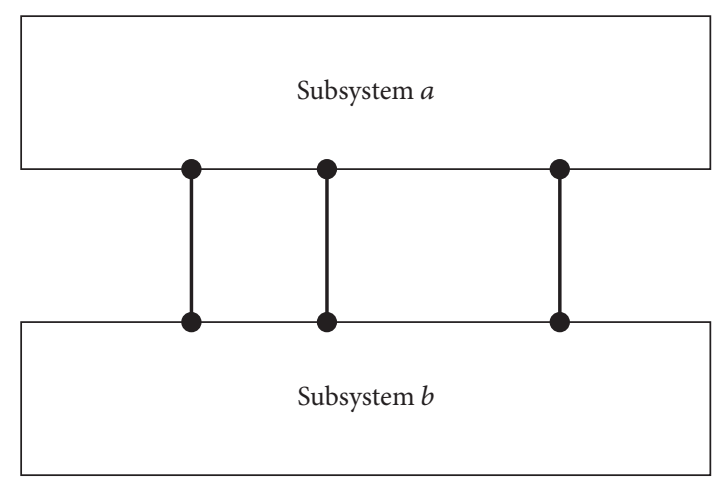

FIgURE 1: Two generic SEA subsystems connected via discrete couplings.

\section{Dynamic Stiffness Couplings between Two SEA Subsystems with Discrete Connections}

In this section, a system model consisting of two generic SEA subsystems with discrete interfaces is set up, as shown in Figure 1. The intermodal dynamic relations between the two sets of subsystem modes can be found by a subsystem modal analysis technique described in [24]. Similar approaches can also be found in $[6,25]$. The main derivation procedure is briefly given as shown below.

2.1. Intermodal Coupling Stiffness Terms for General Interfaces. First, describe each subsystem by the modes when the subsystem is uncoupled from the rest. For subsystem $a$, for instance, let $\phi_{j}^{a}, \omega_{j}^{a}$, and $\eta_{j}^{a}$ represent the $j$ th mass-normalized mode shape, the corresponding natural frequency, and modal loss factor, respectively. The modal receptance matrix of subsystem $a, \mathbf{Y}^{\mathbf{a}}$, which is diagonal, can then be expressed as

$$
Y_{j j}^{a}=\frac{1}{\left(\omega_{j}^{a}\right)^{2}\left(1+i \eta_{j}^{a}\right)-\omega^{2}} .
$$

In $(1) i^{2}=-1$. Clearly there exists $Y_{j j}^{a}=q_{j}^{a} / f_{j}^{a}$, where $q_{j}^{a}$ and $f_{j}^{a}$ are the $j$ th modal amplitude and modal force of the subsystem, respectively. For subsystem $b$, similar expressions can be established by replacing the superscript $a$ with $b$ and subscript $j$ with $k$.

Assume that the interface forces and displacements can be decomposed along the interface region $\sigma_{I}$ in terms of a set of interface basis functions $\psi\left(\sigma_{I}\right)$, that is,

$$
\begin{aligned}
F_{I}\left(\sigma_{I}\right) & =\sum_{p} f_{I, p} \psi_{p}\left(\sigma_{I}\right), \\
W_{I}\left(\sigma_{I}\right) & =\sum_{p} q_{I, p} \psi_{p}\left(\sigma_{I}\right),
\end{aligned}
$$

where $p$ is the order of the basis function, and

$$
\int_{\sigma_{I}} \psi_{p}\left(\sigma_{I}\right) \psi_{p^{\prime}}\left(\sigma_{I}\right) \mathrm{d} \sigma_{I}=\delta_{p p^{\prime}}
$$


where $\delta$ is Dirac delta function. Such an interface decomposition technique has been found to be particularly useful when dealing with continuous interfaces [25].

If the modes in subsystems $a$ and $b$ as well as the interface modes can be truncated into finite sets, by making use of the modal orthogonal properties, both the interface modal force, $f_{I, p}$, and the interface modal amplitude, $q_{I, p}$, can be expressed in terms of the sets of modal forces, $f_{I}^{a}$ and $f_{I}^{b}$, as well as the sets of modal amplitudes $q_{I}^{a}$ and $q_{I}^{b}$. By enforcing force equilibrium and displacement continuity boundary conditions along the interface between subsystems $a$ and $b$, the modal amplitudes of each subsystem can finally be calculated as [24]

$$
\left\{\begin{array}{l}
\mathbf{q}^{\mathbf{a}} \\
\mathbf{q}^{\mathbf{b}}
\end{array}\right\}=\left[\begin{array}{cc}
\mathrm{G}^{\mathrm{aa}} & \mathrm{G}^{\mathrm{ab}} \\
\mathbf{G}^{\mathbf{a b}^{T}} & \mathrm{G}^{\mathbf{b b}}
\end{array}\right]\left\{\begin{array}{c}
\mathbf{f}_{\mathrm{e}}^{\mathrm{a}} \\
\mathbf{f}_{\mathrm{e}}^{\mathbf{b}}
\end{array}\right\} .
$$

In (5), the superscript $\mathrm{T}$ represents transpose and $\mathbf{q}^{\mathbf{a}}$ and $\mathbf{f}_{\mathbf{e}}^{\mathbf{a}}$ are both column vectors whose $j$ th elements $q_{j}^{a}$ and $f_{e, j}^{a}$ represent, respectively, the $j$ th modal amplitude and modal force of subsystem $a$ corresponding to the external excitation $F_{e}^{a}$ at $\sigma_{e}^{a}$, of which

$$
f_{e, j}^{a}=\int_{\sigma_{a}} \phi_{j}^{a}\left(\sigma_{e}^{a}\right) F_{e}^{a}\left(\sigma_{e}^{a}\right) \mathrm{d} \sigma_{e}^{a}
$$

Similar expressions can also be established for subsystem $b$ by replacing the superscript $a$ with $b$, and subscript $j$ with $k$, as appropriate.

$\mathbf{G}^{\mathbf{a a}}$ and $\mathbf{G}^{\mathbf{a b}}$ are, respectively, the Green function matrices which give the modal amplitudes of subsystem $a$ upon per unit modal forces applied on the subsystem itself and the modal amplitudes of subsystem $a$ upon per unit modal forces applied on subsystem $b$. These Green function matrices, after some algebraic, can be determined by

$$
\begin{aligned}
& \mathbf{G}^{\mathbf{a} a}=\mathbf{Y}^{\mathbf{a}}\left[\mathbf{I}-\boldsymbol{\alpha}(\mathbf{A}+\mathbf{B})^{-1} \boldsymbol{\alpha}^{\mathrm{T}} \mathbf{Y}^{\mathbf{a}}\right], \\
& \mathbf{G}^{\mathbf{b} b}=\mathbf{Y}^{\mathbf{b}}\left[\mathbf{I}-\boldsymbol{\beta}(\mathbf{A}+\mathbf{B})^{-1} \boldsymbol{\beta}^{\mathrm{T}} \mathbf{Y}^{\mathbf{b}}\right], \\
& \mathbf{G}^{\mathbf{a b}}=\mathbf{Y}^{\mathbf{a}}\left[\boldsymbol{\alpha}(\mathbf{A}+\mathbf{B})^{-1} \boldsymbol{\beta}^{\mathrm{T}}\right] \mathbf{Y}^{\mathbf{b}},
\end{aligned}
$$

where $\boldsymbol{\alpha}$ and $\boldsymbol{\beta}$ are matrices whose $(j, p)$ th and $(k, p)$ th elements are given by

$$
\begin{aligned}
& \alpha_{j p}=\int_{\sigma_{I}} \phi_{j}^{a}\left(\sigma_{I}\right) \psi_{p}\left(\sigma_{I}\right) \mathrm{d} \sigma_{I}, \\
& \beta_{k p}=\int_{\sigma_{I}} \phi_{k}^{b}\left(\sigma_{I}\right) \psi_{p}\left(\sigma_{I}\right) \mathrm{d} \sigma_{I}
\end{aligned}
$$

while $\mathbf{A}$ and $\mathbf{B}$ are square matrices, given by

$$
\begin{aligned}
& \mathbf{A}=\boldsymbol{\alpha}^{\mathrm{T}} \mathbf{Y}^{\mathbf{a}} \boldsymbol{\alpha}, \\
& \mathbf{B}=\boldsymbol{\beta}^{\mathrm{T}} \mathbf{Y}^{\mathbf{b}} \boldsymbol{\beta} .
\end{aligned}
$$

Physically, A (11) and B (12) represent, respectively, the receptance matrices of subsystem $a$ and $b$ at their interfaces.
$(\mathbf{A}+\mathbf{B})^{-1}$ is therefore the so-called "interface dynamic stiffness matrix" of subsystems $a$ and $b$, determined by the subsystem modal properties as well as their local mode shapes at the interfaces.

From (5), a dynamic stiffness matrix can be obtained as

$$
\left[\begin{array}{cc}
D^{a a} & -D^{a b} \\
-D^{a^{T}} & D^{b b}
\end{array}\right]=\left[\begin{array}{cc}
G^{a a} & G^{a b} \\
G^{a b^{T}} & G^{b b}
\end{array}\right]^{-1},
$$

where

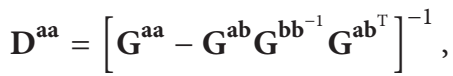

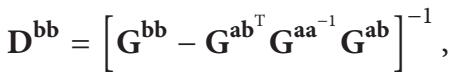

$$
\begin{aligned}
& D^{a b}=D^{a \mathbf{a}} G^{a b} G^{b^{-1}} \text {. }
\end{aligned}
$$

Physically, the $(j, k)$ th entry in $\mathbf{D}^{\mathbf{a b}}$ (16) represents the coupling stiffness between the $j$ th mode of subsystem $a$ and the $k$ th mode of subsystem $b$, while the $\left(j, j^{\prime}\right)$ th entry in $\mathbf{D}^{\text {aa }}$ (14) represents that between the $j$ th and $j^{\prime}$ th modes of subsystem $a$. For convenience, here, these intermodal couplings are termed, respectively, as "out-of-set" and "inset" mode couplings [2]. More discussions on the distinctions of the out-of-set and in-set mode coupling terms can be found in Section 2.2.1.

2.2. Intermodal Coupling Stiffness Terms for Discrete Interfaces. Assume the discrete interfaces composed of $N$ coupling points. By the criterion of (4), the interface basis functions in (2) here can then be chosen as a unit diagonal matrix with a size of $N$. (Note that, in general, there should be many choices of the sets of basis functions provided they are complete and orthogonal and can represent the interface deformations between the two subsystems well [25]; usually the set of local modal shapes defined on the interface DOFs can be a better choice of the basis functions [24]). Consequently, (10) simply yields

$$
\begin{aligned}
& \alpha_{j p}=\phi_{j}^{a}\left(\sigma_{I, p}\right), \quad p=1, \ldots, N, \\
& \beta_{k p}=\phi_{k}^{b}\left(\sigma_{I, p}\right), \quad p=1, \ldots, N .
\end{aligned}
$$

Substituting (17) into (11) and (12), the ( $\left.p, p^{\prime}\right)$ th elements of matrices $\mathbf{A}$ and $\mathbf{B}$ can thus be obtained, respectively, as

$$
\begin{aligned}
& A_{p p^{\prime}}=\sum_{j} \frac{\phi_{j}^{a}\left(\sigma_{I, p}\right) \phi_{j}^{a}\left(\sigma_{I, p^{\prime}}\right)}{\left(\omega_{j}^{a}\right)^{2}\left(1+i \eta_{j}^{a}\right)-\omega^{2}} \\
& B_{p p^{\prime}}=\sum_{k} \frac{\phi_{k}^{b}\left(\sigma_{I, p}\right) \phi_{k}^{b}\left(\sigma_{I, p^{\prime}}\right)}{\left(\omega_{k}^{b}\right)^{2}\left(1+i \eta_{k}^{b}\right)-\omega^{2}} .
\end{aligned}
$$

It is seen that $A_{p p^{\prime}}$ is exactly the receptance of subsystem $a$ between its connecting points $\sigma_{I, p}$ and $\sigma_{I, p^{\prime}}$, that is, $A_{p p^{\prime}}=$ $Y_{p p^{\prime}}^{a}$. Similarly, $B_{p p^{\prime}}=Y_{p p^{\prime}}^{b}$. Therefore, $\mathbf{A}$ and $\mathbf{B}$ are actually 
the receptance matrices of subsystems $a$ and $b$ at their interfaces.

If it is assumed that the interface degrees of freedom act independently so that the coherences among the interface points are ignorable, then by (18), $\mathbf{A}$ and $\mathbf{B}$ tend to be diagonally dominated so that

$$
[A+B]_{p p} \approx Y_{p p}^{a}+Y_{p p}^{b} .
$$

Note that (19) is usually true if both subsystems have high modal densities and the interface points are spaced at least one wavelength apart from each other [26]. As a result, $\mathbf{G}_{\mathbf{a a}}$ and $\mathbf{G}_{\mathbf{b b}}$, by (7) and (8), become diagonally dominated as well with

$$
\begin{aligned}
G_{j j}^{a a} & \approx Y_{j j}^{a}[1 \\
& \left.-\sum_{p}\left(\frac{1}{Y_{p p}^{a}+Y_{p p}^{b}} \frac{\phi_{j}^{a}\left(\sigma_{I, p}\right) \phi_{j}^{a}\left(\sigma_{I, p}\right)}{\left(\omega_{j}^{a}\right)^{2}\left(1+i \eta_{j}^{a}\right)-\omega^{2}}\right)\right], \\
G_{k k}^{b b} & \approx Y_{k k}^{b}[1 \\
& \left.-\sum_{p}\left(\frac{1}{Y_{p p}^{a}+Y_{p p}^{b}} \frac{\phi_{k}^{b}\left(\sigma_{I, p}\right) \phi_{k}^{b}\left(\sigma_{I, p}\right)}{\left(\omega_{k}^{b}\right)^{2}\left(1+i \eta_{k}^{b}\right)-\omega^{2}}\right)\right] .
\end{aligned}
$$

For SEA subsystems with high modal densities, the second terms on the brackets of the right sides in (20) tend to be much less than unity. It thus implies that the Green functions of each SEA subsystem can only be slightly affected by the other through the couplings. In which case, (9) leads to

$$
G_{j k}^{a b} \approx Y_{j j}^{a}\left[\sum_{p} \frac{\phi_{j}^{a}\left(\sigma_{I, p}\right) \phi_{k}^{b}\left(\sigma_{I, p}\right)}{(A+B)_{p p}}\right] Y_{k k}^{b} .
$$

Substituting (20)-(21) into (14)-(16), the dynamic coupling stiffness between any two pair of subsystem modes can be simply determined as

$$
D_{j k}^{a b} \approx \sum_{p} \frac{\phi_{j}^{a}\left(\sigma_{I, p}\right) \phi_{k}^{b}\left(\sigma_{I, p}\right)}{(A+B)_{p p}} .
$$

Considering the diagonal properties of matrices $\mathbf{A}$ and $\mathbf{B},(22)$ then becomes

$$
D_{j k}^{a b} \approx \sum_{p} \phi_{j}^{a}\left(\sigma_{I, p}\right) K_{p p}^{a b} \phi_{k}^{b}\left(\sigma_{I, p}\right),
$$

where,

$$
K_{p p}^{a b}=\left(Y_{p p}^{a}+Y_{p p}^{b}\right)^{-1} \approx\left(Y_{\mathrm{in}, \infty}^{a}+Y_{\mathrm{in}, \infty}^{b}\right)^{-1}
$$

where $Y_{\text {in, } \infty}^{a}$ and $Y_{\text {in, } \infty}^{b}$ are the characteristic receptances of the two subsystems when the subsystems are extended to infinity. Equation (23) can then be further reduced to

$$
D_{j k}^{a b} \approx\left(Y_{\mathrm{in}, \infty}^{a}+Y_{\mathrm{in}, \infty}^{b}\right)^{-1} \sum_{p} \phi_{j}^{a}\left(\sigma_{I, p}\right) \phi_{k}^{b}\left(\sigma_{I, p}\right) .
$$

Equation (25) indicates that the intermodal coupling stiffness term $D_{j k}^{a b}$ is a frequency-dependent function, determined by the characteristic receptances of the two subsystems $Y_{\mathrm{in}, \infty}^{a}$ and $Y_{\mathrm{in}, \infty}^{b}$ as well as the mode shapes of the two subsystems $\phi_{j}^{a}$ and $\phi_{k}^{b}$ at their interfaces $\sigma_{I}$. Moreover, as the mean of $D_{j k}^{a b}$ is mainly determined by $\left(Y_{\mathrm{in}, \infty}^{a}+Y_{\mathrm{in}, \infty}^{b}\right)$, the variance of $D_{j k}^{a b}$ is mainly determined by those of $\phi_{j}^{a}$ and $\phi_{k}^{b}$ at the interfaces. In the contents below, (25) will be firstly used in a coupling case with single-point connection in order to demonstrate its application.

2.2.1. Application to a Single-Point Coupling Case. For a single-point coupling between subsystems $a$ and $b$, the interface function can be simply defined as $\psi_{p}\left(\sigma_{I}\right)=1$ with $p=1$. A and B (see (11) and (12)) are simply the inputreceptances of the two subsystems at their connecting points $\sigma_{I}^{a}$ and $\sigma_{I}^{b}$, as

$$
\begin{aligned}
& A=Y_{a}\left(\sigma_{I}^{a}\right)=\sum_{j}\left[\phi_{j}^{a 2}\left(\sigma_{I}^{a}\right) Y_{j j}^{a}\right], \\
& B=Y_{b}\left(\sigma_{I}^{b}\right)=\sum_{k}\left[\phi_{k}^{b 2}\left(\sigma_{I}^{b}\right) Y_{k k}^{b}\right] .
\end{aligned}
$$

It thus gives (by (24))

$$
K_{a b}=\frac{1}{Y_{a}\left(\sigma_{I}^{a}\right)+Y_{b}\left(\sigma_{I}^{b}\right)} \approx \frac{1}{Y_{\mathrm{in}, \infty}^{a}+Y_{\mathrm{in}, \infty}^{b}} .
$$

Then, (7)-(9) become

$$
\begin{aligned}
& \mathbf{G}^{\mathbf{a} \mathbf{a}}=\mathbf{Y}^{\mathbf{a}}\left[\mathbf{I}-K_{a b} \Phi^{\mathbf{a}} \Phi^{\mathbf{a}^{\mathrm{T}}} \mathbf{Y}^{\mathbf{a}}\right], \\
& \mathbf{G}^{\mathbf{b} \mathbf{b}}=\mathbf{Y}^{\mathbf{b}}\left[\mathbf{I}-K_{a b} \Phi^{\mathbf{b}} \Phi^{\mathbf{b T}} \mathbf{Y}^{\mathbf{b}}\right], \\
& \mathbf{G}^{\mathbf{a b}}=K_{a b} \mathbf{Y}^{\mathbf{a}} \boldsymbol{\Phi}^{\mathbf{a}} \boldsymbol{\Phi}^{\mathbf{b T}} \mathbf{Y}^{\mathbf{b}} .
\end{aligned}
$$

Equations (28)-(30) can then be combined with (13) to determine both the in-set $\left(\mathbf{D}^{\mathbf{a} \mathbf{a}}\right.$ and $\left.\mathbf{D}^{\mathbf{b} \mathbf{b}}\right)$ and out-of-set $\left(\mathbf{D}^{\mathbf{a b}}\right)$ intermodal coupling stiffness terms.

In the sense of weak coupling, that is, the second terms in brackets of the right sides of (28) and (29) are both negligible [14], the coupling stiffness between the $j$ th mode of subsystem $a$ and the $k$ th mode of subsystem $b$, by (16), thus gives

$$
D_{j k}^{a b}=\phi_{j}^{a}\left(\sigma_{I}^{a}\right) K_{a b} \phi_{k}^{b}\left(\sigma_{I}^{b}\right) .
$$

In [2], the intermodal coupling stiffness, for two subsystems connected by a single spring $K$, is given by

$$
D_{j k}^{a b} \approx \phi_{j}^{a}\left(x_{I}^{a}\right) K \phi_{k}^{b}\left(x_{I}^{b}\right) .
$$

It is seen that (31) and (32) are in a very similar form, except that $K_{a b}$ is a frequency-dependent function in the former equation while $K$ is a constant in the latter one. It thus allows the results in [2] for spring-coupled subsystems to be extended for the coupling cases with rigid-point connections in a way fairly straightforward. These are described in the section below. 


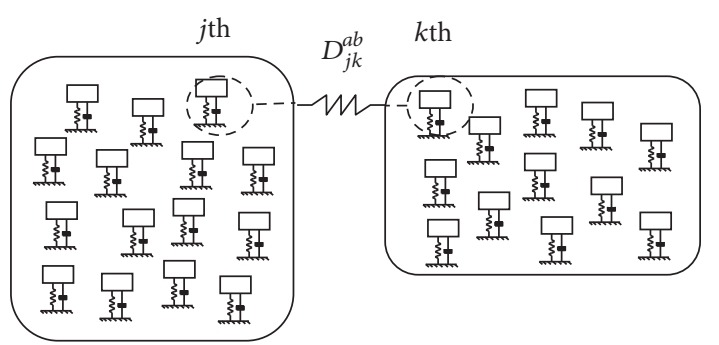

FIgURE 2: Two sets of oscillators connected via a set of stiffness.

\section{Formulas of CLF Estimation and Coupling Strength Measurement}

In [2], expressions of CLF estimations and coupling strength measurement were presented for two sets of oscillators coupled via a set of spring stiffness, as shown in Figure 2, based on the statistical power-energy relations between the two subsystems. These are first briefly reviewed in Section 3.1, and then extensions are made in Section 3.2 for the rigidpoint coupling cases.

3.1. Formulas for Spring-Connected Subsystems. For simplicity, both subsystems are assumed to be uniform and homogenous so that both the modal masses $\left(m_{a}\right.$ and $\left.m_{b}\right)$ and modal loss factors $\left(\eta_{a}\right.$ and $\left.\eta_{b}\right)$ are constant.

Let $D_{j k}^{a b}$ represent the coupling stiffness between the $j$ th oscillator in set $a$ and the $k$ th oscillator in set $b$. The coupling strength between the two sets of spring-coupled oscillators can be measured by a so-called "coupling strength parameter" $\gamma^{2}$ [1], which was given in [2] in the form of

$$
\gamma^{2}=\frac{E\left[\left|D_{j k}^{a b}\right|^{2}\right]}{m_{a} m_{b} \eta_{a} \eta_{b} \omega^{4}}
$$

where $\omega$ is the centre frequency of the frequency band of interest, $n_{a}$ and $n_{b}$ are the modal densities of subsystems $a$ and $b$, and $E[\cdot]$ represents the expectation over the ensemble. The weak coupling region between the two subsystems corresponds to [1].

$$
\gamma^{2} \ll 1
$$

In case of weak coupling (i.e. (34) is satisfied), the coupling loss factors $\eta_{a b}$ and $\eta_{b a}$ for the two subsystems $a$ and $b$ can then be estimated as [2]

$$
n_{a} \eta_{a b}=n_{b} \eta_{b a} \approx \frac{\pi}{2} \frac{n_{a} n_{b}}{m_{a} m_{b} \omega^{3}} E\left[\left|D_{j k}^{a b}\right|^{2}\right]
$$

For a single-spring coupling $K$ between $a$ and $b$, for example, $D_{j k}^{a b}$ can be derived as (32). Substituting (32) into (35), $\eta_{a b}$ and $\eta_{b a}$ can then be calculated in a very simple manner [2]. In the subsection below, it will show how (33) and (35) can be extended readily to the coupling cases with multipoint interfaces.
3.2. Formulas for Rigid-Point Connections. For rigid-point couplings between $a$ and $b, D_{j k}^{a b}$ (given by (25)) becomes a frequency-dependent complex variable. Equations (33) and (35) thus need to be modified as

$$
\begin{aligned}
\gamma^{2} & =\frac{E\left[\left|\bar{D}_{j k}^{a b}\right|^{2}\right]}{m_{a} m_{b} \eta_{a} \eta_{b} \omega^{4}}, \\
n_{a} \eta_{a b} & =n_{b} \eta_{b a} \approx \frac{\pi}{2} \frac{n_{a} n_{b}}{m_{a} m_{b} \omega^{3}} E\left[\left|\bar{D}_{j k}^{a b}\right|^{2}\right],
\end{aligned}
$$

where ${ }^{-}$represents the expectation over the frequency band of interest.

Considering the independence of $\phi_{j}^{a}, \phi_{k}^{b}$, and $K_{p p}^{a b}$, leads to

$$
\begin{aligned}
& E\left[\left|\bar{D}_{j k}^{a b}\right|^{2}\right] \\
& \quad \approx \sum_{p} E\left[\left|\phi_{j}^{a}\left(\sigma_{p}^{a}\right)\right|^{2}\right] E\left[\left|\bar{K}_{p p}^{a b}\right|^{2}\right] E\left[\left|\phi_{k}^{b}\left(\sigma_{p}^{b}\right)\right|^{2}\right] .
\end{aligned}
$$

If $\phi_{j}^{a}$ and $\phi_{k}^{b}$ are chosen to be mass-normalized, there exist

$$
\begin{aligned}
& E\left[\left|\phi_{j}^{a}\left(\sigma_{p}^{a}\right)\right|^{2}\right]=\frac{1}{M_{a}}, \\
& E\left[\left|\phi_{k}^{b}\left(\sigma_{p}^{b}\right)\right|^{2}\right]=\frac{1}{M_{b}}
\end{aligned}
$$

where $M_{a, b}$ are the total masses of subsystems $a$ and $b$. Substituting (39) into (38) and considering (24) yield

$$
E\left[\left|\bar{D}_{j k}^{a b}\right|^{2}\right] \approx \frac{N}{M_{a} M_{b}} \frac{1}{\left|Y_{\mathrm{in}, \infty}^{a}+Y_{\mathrm{in}, \infty}^{b}\right|^{2}} .
$$

The above equation shows that $E\left[\left|\bar{D}_{j k}^{a b}\right|^{2}\right]$ is determined by the characteristic dynamic properties and the total mass of each subsystem as well as the number of coupling points between them regardless of the subsystem modal properties (modal mass, modal density, and modal damping). It thus indicates that the statistics of the dynamic coupling stiffness terms between two sets of subsystem modes are independent of the exact modal properties of each subsystem.

Substituting (40) into (36) and considering $m_{a}=m_{b}=$ 1 (mass-normalized mode shapes), the coupling strength parameter can finally be expressed as

$$
\gamma^{2} \approx \frac{N}{M_{a} M_{b} \eta_{a} \eta_{b} \omega^{4}}\left(\frac{1}{\left|Y_{\mathrm{in}, \infty}^{a}+Y_{\mathrm{in}, \infty}^{b}\right|^{2}}\right) .
$$

The weak coupling region of two discretely connected subsystems can then be distinguished simply and explicitly by $\gamma^{2} \ll 1$. As a result, the low bound of the frequency range of weak coupling region can be determined as

$$
\omega_{\text {weak }}^{2} \gg \frac{1}{\left|Y_{\mathrm{in}, \infty}^{a}+Y_{\mathrm{in}, \infty}^{b}\right|} \sqrt{\frac{N}{M_{a} M_{b} \eta_{a} \eta_{b}}} .
$$


For the frequency range where (42) is satisfied, the CLFs of two subsystems with discrete rigid-point couplings, by substituting (40) into (37), can be estimated as

$$
n_{a} \eta_{a b}=n_{b} \eta_{b a} \approx N \frac{\pi}{2} \frac{n_{a} n_{b}}{M_{a} M_{b} \omega^{3}} \frac{1}{\left|Y_{\mathrm{in}, \infty}^{a}+Y_{\mathrm{in}, \infty}^{b}\right|^{2}} .
$$

Equation (43) is expected to be valid if (42) (weak coupling) is satisfied and also the coherences among the coupling points are ignorable which commonly requires that coupling points are located at least one wavelength apart from each other [26]. A good supporting evidence is given here in the first instance to show the validity of (43).

3.2.1. A Special Case of Single-Point Coupling. For a singlepoint connection between subsystems $a$ and $b,(43)$ gives

$$
\omega n_{a} \eta_{a b} \approx \frac{\pi}{2} \frac{n_{a} n_{b}}{M_{a} M_{b} \omega^{2}} \frac{1}{\left|Y_{\mathrm{in}, \infty}^{a}+Y_{\mathrm{in}, \infty}^{b}\right|^{2}} .
$$

Another well-known CLF expression is available in [1] (by substituting (10.1.3) into (10.1.6) in [1]), as

$$
\omega n_{a} \eta_{a b} \approx \frac{1}{2 \pi}\left(\frac{4 \operatorname{Re}\left\{Z_{\infty}^{a}\right\} \operatorname{Re}\left\{Z_{\infty}^{b}\right\}}{\left|Z_{\infty}^{a}+Z_{\infty}^{b}\right|^{2}}\right),
$$

where $Z_{\infty}^{a}$ represents the characteristic impedance of subsystem $a$, by $[1]$

$$
\begin{gathered}
Z_{\infty}^{a}=\frac{1}{\left(i \omega Y_{\mathrm{in}, \infty}^{a}\right)} \\
\operatorname{Re}\left\{Z_{\infty}^{a}\right\}=\omega\left|Y_{\mathrm{in}, \infty}^{a}\right| \approx \frac{\pi n_{a}}{2 M_{a}} .
\end{gathered}
$$

$Z_{\infty}^{b}$ and $\operatorname{Re}\left\{Z_{\infty}^{b}\right\}$ can be determined similarly by replacing the superscript $a$ to $b$ in (46). Substituting (46) into (45), it generates exactly the form of (44).

Note that (45) was derived in [1] by using the wave approach and transmission coefficient; what (44) proposed here was derived by using the modal approach and intermodal couplings. The good consistency between (44) and (45) thus strongly supports the validity of the derivation procedure of (43).

As stated previously in Section 1 that, apart from (10.1.6), a CLF formula for the single-point connection proposed by a subsystem modal approach and the transmission coefficients is also available in [1] (i.e. (10.1.18), p. 188). However, because both (10.1.6) and (10.1.18) were developed by using onedimensional subsystems and incoherent waves and then assumed (without being proven) to be applicable for twoand three-dimensional subsystems, one may become quite hesitated to extend directly (10.1.6) or (10.1.18) to coupling cases with many interface degrees of freedom in that more implicit and/or explicit assumptions have to be introduced and involved. Here the derivation of (43) has just proved that (10.1.6) or (10.1.18) can be extended to the multidegrees of freedom coupling cases but by using intermodal couplings

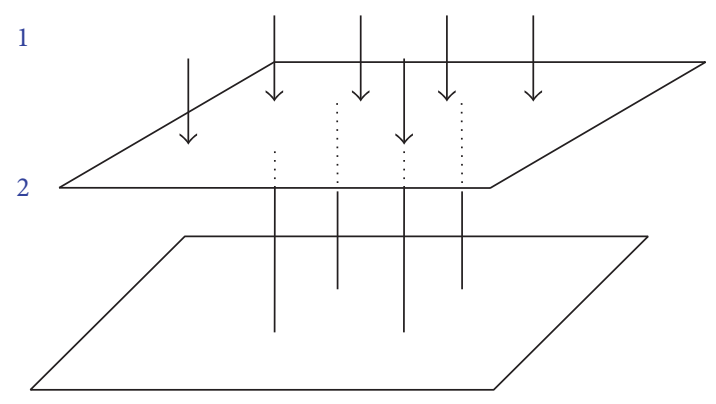

FIGURE 3: Two plates connected via discrete points.

rather than the wave transmission coefficients. Nevertheless, comparing (44) with (10.1.18) in [1], it can be seen clearly that the intermodal coupling-based solution has a much simpler form than the transmission-coefficient-based one, although the two expressions should be closely related to each other.

3.3. Result Discussion. From the analysis given in the above subsection, it is seen that (1) subsystem damping loss factors have strong influence on the coupling strength (41) but have little effect on the CLFs (43) which is in good agreement with the existing SEA literature; (2) both coupling strength and coupling loss factor are proportional to $N$ but inversely proportional to $M_{a}, M_{b}$, and $\left|Y_{\mathrm{in}, \infty}^{a}+Y_{\mathrm{in}, \infty}^{b}\right|^{2}$; and (3) the coupling strength seems affected more by frequency than by the CLFs.

If it is assumed that $M_{a} \approx M_{b} \approx M$, considering $\left|Y_{\mathrm{in}, \infty}^{a}\right| \approx$ $\pi n_{a} / 2 M_{a} \omega$ [1], (41) and (42) can be further simplified as

$$
\left(\Delta_{a}+\Delta_{b}\right) \gg \frac{2}{\pi} \sqrt{N},
$$

where, $\Delta_{a}$ and $\Delta_{b}$ are, respectively, the modal overlap factors of subsystems $a$ and $b$. Equation (47) thus explains well that weak couplings tend to occur if both subsystems have high modal overlaps. Although being derived under the condition of $M_{a} \approx M_{b} \approx M$, (47) can be reasonably taken as valid for more general coupling cases in that it makes a good sense of physical intuitive.

It is worth noting here that (47) implies that weak coupling may not necessarily require each individual subsystem to be with a high modal overlap factor. Instead, it may only need the summation of the subsystem modal overlap factors to be big enough. It thus makes it possible to extend the conventional SEA modelling to the mid-frequency range where one or two subsystems might with a quite low modal overlap factor $[24,27]$. Further relevant investigations are underway [28].

\section{Numerical Examples}

In this section, numerical examples are given to illustrate the performance of the two new formulas (see (41) and (43)) for discretely connected subsystems.

The numerical model employed is shown in Figure 3, which is comprised of two plates connected by rigid points. 
The number of coupling points considered is varied between 1 and 4, and both "large"- and "small"-spacing cases are involved. (Large spacing means the points are spaced at least one wavelength apart for the frequency range of interest, while small spacing is less than one wavelength apart). For each coupling case, coupling loss factors between the two plates are first calculated by (43) and then compared with the results generated from the PIM method. Here, the PIM-based results are obtained from the ensemble average of the CLFs of 30 samples of the plate-plate models. For each sample, both plates have fixed modal densities and total masses, while their natural frequencies and mode shapes may differ from one to another. This is achieved by varying the ratio of the length and width of each plate model while keeping its area unchanged ( $\pm 5 \%$ of the nominal value). Frequency-averaged energy response and power transmission for each sample of the ensemble under randomly located force excitations are first calculated by exact modal approach, and then ensemble averages are taken over the whole 30 samples. Coupling loss factors can finally be estimated, by its definition given in [1], from the frequency- and ensemble-averaged powerenergy relations between the two plates. The performance of the two new formulas (see (41) and (43)) can then be evaluated by comparing with the PIM-based results. Only the bending motions of the numerical model are considered in the following calculations.

4.1. System Model Description. In Figure 3, both plates 1 and 2 are rectangular with simply supported edges. Plate 1 has an area of $0.63 \mathrm{~m}^{2}$ while plate 2 has an area of $0.8 \mathrm{~m}^{2}$, and both are with the same thickness of $2 \mathrm{~mm}$. The material of the plates is chosen to be steel with Young's modulus of $2.1 \times 10^{11} \mathrm{~N} / \mathrm{m}^{2}$, density of $7.85 \times 10^{3} \mathrm{~kg} / \mathrm{m}^{3}$, Poisson ratio of 0.3 , and damping loss factor of 0.01 . The corresponding modal densities of plates 1 and 2 are $0.016 \mathrm{modes} / \mathrm{rad} / \mathrm{s}$ and $0.02 \mathrm{modes} / \mathrm{rad} / \mathrm{s}$, respectively, and the characteristic impedances $Z_{\infty}^{(1)}=Z_{\infty}^{(2)}=393.2 \mathrm{~N} / \mathrm{m} / \mathrm{s}$.

The frequency range considered is up to $2000 \mathrm{~Hz}$ which comprises approximately 200 resonant modes of plate 1 and 250 resonant modes of plate 2 . The plate wavelength at $500 \mathrm{~Hz}$ is about $0.2 \mathrm{~m}$ while the wavelength at $2000 \mathrm{~Hz}$ is about $0.1 \mathrm{~m}$. The coupling points on plates 1 and 2 are all randomly chosen over surface of each plate but deliberately kept to be spaced at least $0.2 \mathrm{~m}$ apart from all the plate edges in order to minimize the boundary effects for the frequencies above $500 \mathrm{~Hz}$.

\section{Results and Discussions}

Figure 4 shows the coupling strength parameter $\gamma^{2}$ calculated by (41) when the plates are connected by 1 point, 2 points, and 4 points, respectively. It is seen that the frequencies corresponding to $\gamma^{2}=1$ for the three coupling cases are about $280 \mathrm{~Hz}, 400 \mathrm{~Hz}$, and $560 \mathrm{~Hz}$, respectively. The weak coupling regions can then be distinguished explicitly by (34), or equivalently, by (42).

Figures 5-7 compare the coupling loss factor results calculated by (43) and by the PIM-based simulations when $N=1,2$, and 4, respectively. Here it should be mentioned

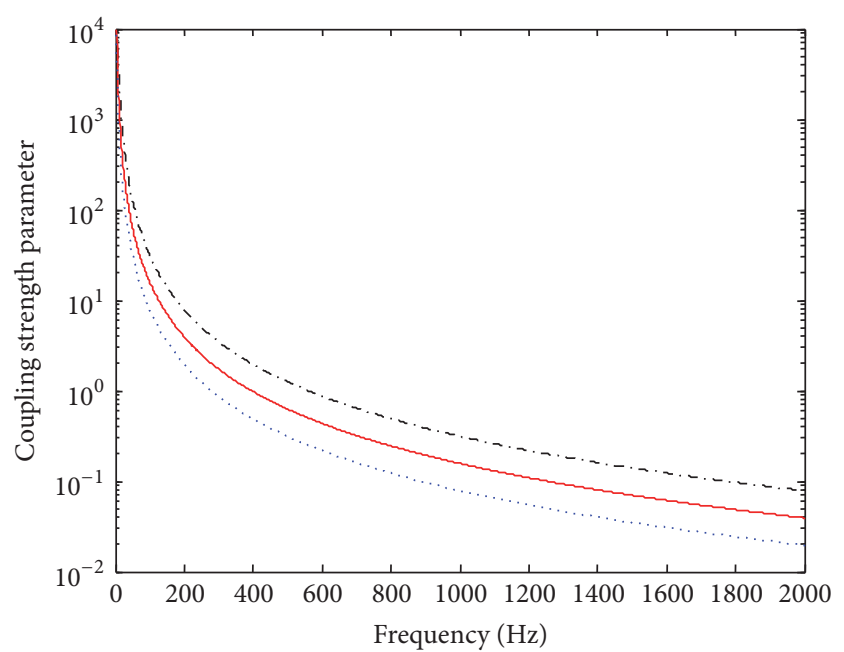

FIGURE 4: Coupling strength parameter $\gamma^{2}$ between two coupled plates: $N=1$ (dotted line); $N=2$ (real line); $N=4$ (dash-dotted line).

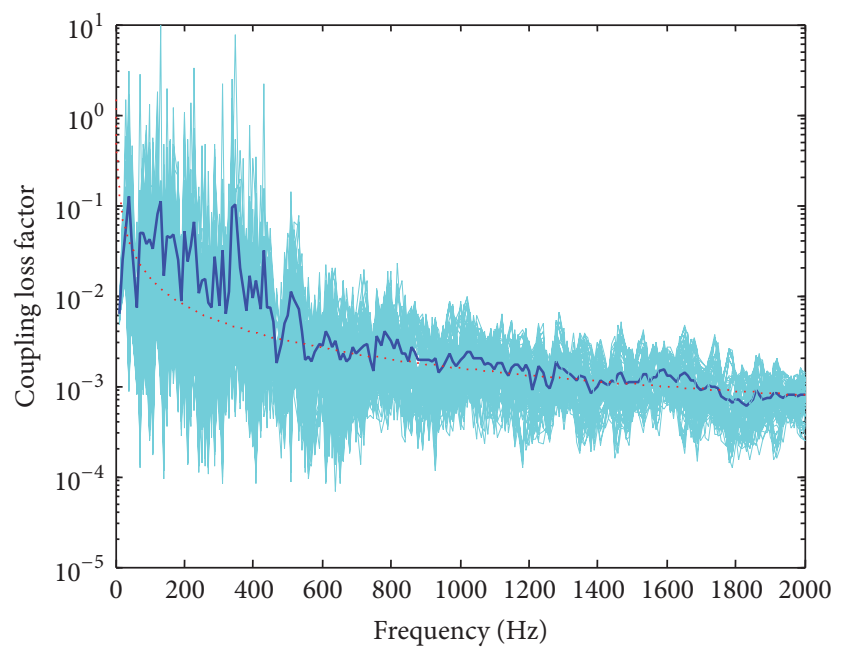

FIGURE 5: Comparison of $\eta_{12}$ when $N=1$ : PIM simulations (real thin lines); ensemble average of the PIM solutions (thick real line); (43) (dotted line).

that, in Figures 6 and 7, the PIM-based CLF solutions correspond to the "widely-spaced" coupling case; that is, the coupling points are located more than $0.2 \mathrm{~m}$ apart from each other so that the coherences between them can be neglected for the frequencies above $500 \mathrm{~Hz}$.

It is seen clearly from Figures 5-7 that the results of (43) tend to agree well with the PIM solutions from the frequency ranges of $\omega \geq 550 \mathrm{~Hz}$ for $N=1$ (Figure 5), $\omega \geq 650 \mathrm{~Hz}$ for $N=2$ (Figure 6), and $\omega \geq 900 \mathrm{~Hz}$ for $N=4$ (Figure 7). It thus indicates that the weak coupling regions start from about $550 \mathrm{~Hz}$ for the case of $N=1$, about $650 \mathrm{~Hz}$ for the case of $N=2$, and about $900 \mathrm{~Hz}$ for the case of $N=4$. It is very interesting to see in Figure 4 that all these weak coupling frequencies correspond to a coupling strength parameter of 


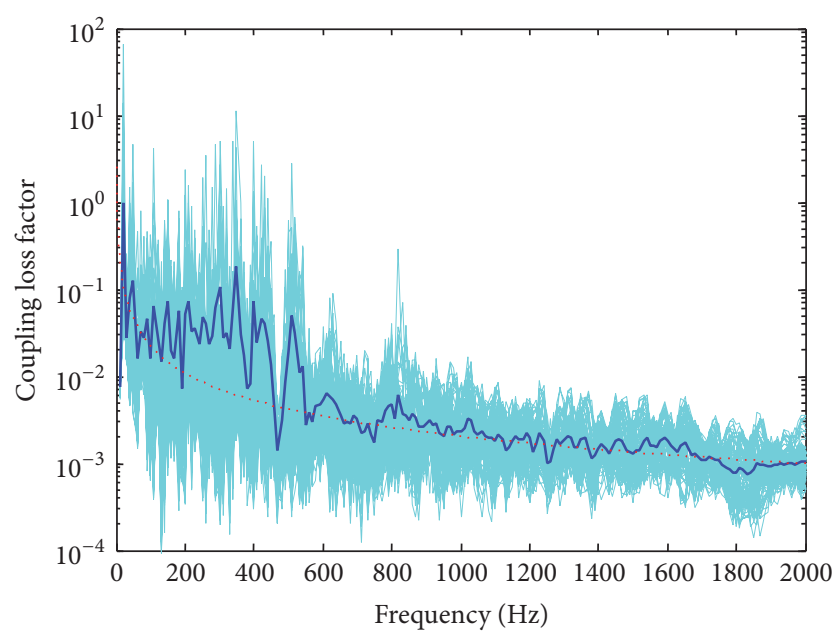

FIgure 6: Comparison of $\eta_{12}$ when $N=2$ (widely-spaced): PIM simulations (real thin lines); ensemble average of the PIM solutions (thick real line); (43) (dotted line).

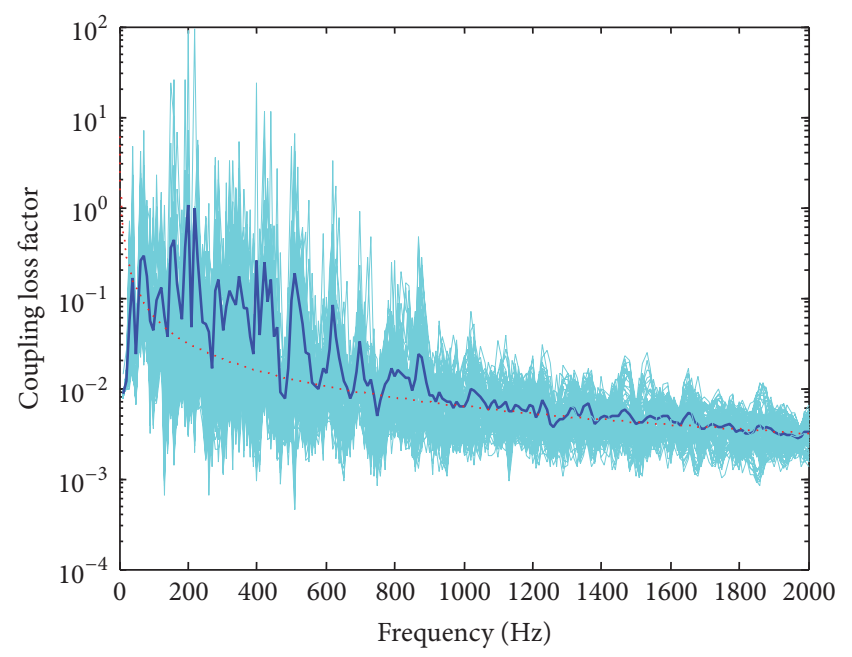

FIgURE 7: Comparison of $\eta_{12}$ when $N=4$ (widely-spaced): PIM simulations (real thin lines); ensemble average of the PIM solutions (thick real line); (43) (dotted line).

$\gamma^{2} \approx 0.3$. It thus implies that (43) is valid for the frequencies where $\gamma^{2} \leq 0.3$ is satisfied, at least for the coupling cases considered in the present investigation.

The apparent fluctuations of the PIM solutions occurring at lower frequencies (below $500 \mathrm{~Hz}$ ) can be explained as the result of insufficient statistical overlap of the ensemble examples [27]. They gradually improve as frequency increases. The poor agreements at lower frequencies are due to the strong coupling effects between the two plates.

Figures 8 and 9 show the comparisons of $\eta_{12}$ calculated by (43) and that by the PIM simulations for the coupling cases of $N=2$ and $N=4$, respectively, of which the coupling points are spaced deliberately at less than $0.1 \mathrm{~m}$ apart from each other, that is, the "narrowly-spaced" couplings. It is seen

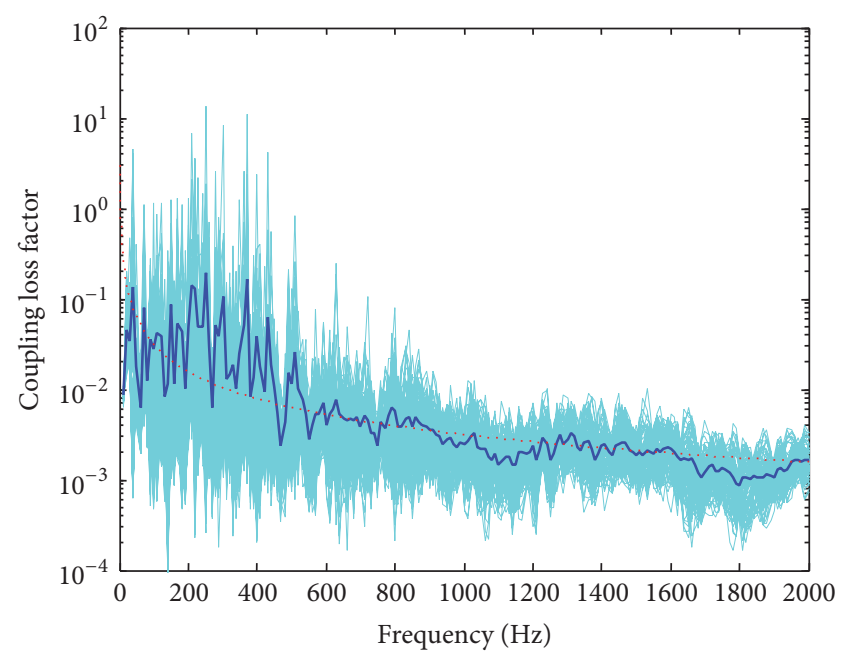

Figure 8: Comparison of $\eta_{12}$ when $N=2$ (narrowly-spaced): PIM simulations (real thin lines); ensemble average of the PIM solutions (thick real line); (43) (dotted line).

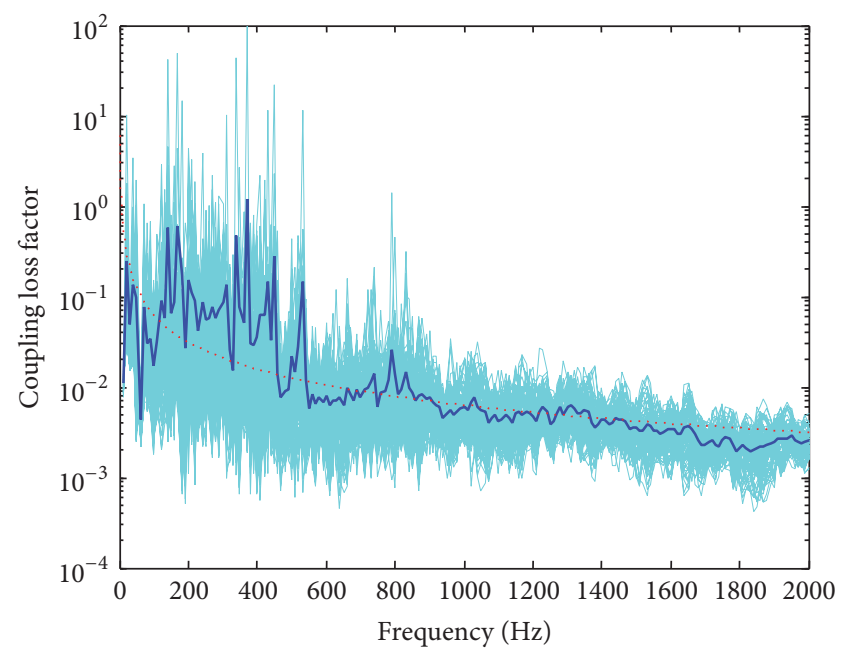

FIGURE 9: Comparison of $\eta_{12}$ when $N=4$ (narrowly-spaced): PIM simulations (real thin lines); ensemble average of the PIM solutions (thick real line); (43) (dotted line).

that the agreements between the two sets of results in Figures 8 and 9 are relatively poor compared to those observed in Figures 6 and 7 for the "widely-spaced" couplings. Clearly this is because (43) was derived by ignoring the coherences among different coupling points, which does not meet when the coupling points are "narrowly-spaced" in Figures 8 and 9. However, it is also seen that (43) works well for certain frequency regions in Figures 8 and 9, such as for the frequency ranges of $600-1000 \mathrm{~Hz}$ and $1200-1700 \mathrm{~Hz}$ in Figure 8 and $900-1700 \mathrm{~Hz}$ in Figure 9, even when the coupling points are closely located with each other. It thus suggests that the coherences between the coupling points may be not so important for certain frequency ranges, at least in the sense of ensemble average. 


\section{Conclusions}

This paper mainly concerns the estimation of coupling loss factors for SEA subsystems connected via discrete interfaces. First of all, the dynamic interactions between two subsystems connected via discrete interfaces are expressed explicitly as an intermodal coupling stiffness matrix by a subsystem modal approach given in [24]. If each subsystem is highly modal dense and each interface degree of freedom is assumed to act independently, the dynamic interactions are found to be strongly dominated by the modal couplings between the two sets of subsystem modes while the couplings within the same set of modes can be neglected. In which case, the modal stiffness coupling terms simply reduce to a function of the characteristic receptances of the two subsystems as well as their mode shapes at the interfaces. If further ensembleand frequency-averaged, these modal coupling terms can be reduced to a function which depends only on the characteristic dynamic properties and the total mass of each subsystem as well as the number of interface degrees of freedom. The results can then be accommodated within the theoretical lines of $[1,2]$ to yield two simple formulas for both coupling strength measurement and coupling loss factor estimation, both depending only on the gross modal properties and the number of coupling points but regardless of any other detailed subsystem modal properties. The consistency and difference of the present CLF formula with and from the traditional CLF solutions in [1] are discussed. Meanwhile, the present theory shows clearly that (1) both coupling strength and CLFs are proportional to the coupling points $N$ and inversely proportional to the total subsystem masses $\left(M_{a}\right.$ and $\left.M_{b}\right)$ and $\left|Y_{\mathrm{in}, \infty}^{a}+Y_{\mathrm{in}, \infty}^{b}\right|^{2}$; (2) the coupling strength is strongly determined by the subsystem damping loss factors while the CLFs are independent of subsystem damping, which are in good agreement with the existing SEA literature; and (3) the coupling strength tends to be more sensitive to the frequencies than the coupling loss factors.

To illustrate the validity and accuracy of the present technique, a few of numerical examples are presented for the plate-plate coupling models with discrete point connections. A very good performance of the present CLF formula is observed when compared with the commonly used power injection method (PIM).

\section{Competing Interests}

The authors declare that there are no competing interests regarding the publication of this paper.

\section{Acknowledgments}

The research is sponsored by the National Natural Science Foundation of China (NSFC, Grants nos. 51175300 and U1434201) and the State Key Laboratory of Vehicle NVH and Safety Technology of China (no. NVHSKL-201503).

\section{References}

[1] R. H. Lyon and R. G. DeJong, Theory and Application of Statistical Energy Analysis, Butterworth, London, UK, 1995.

[2] B. R. Mace and L. Ji, "The statistical energy analysis of coupled sets of oscillators," Proceedings of the Royal Society A: Mathematical, Physical \& Engineering Sciences, vol. 463, no. 2081, pp. 1359-1377, 2007.

[3] S. Finnveden, "A quantitative criterion validating coupling power proportionality in statistical energy analysis," Journal of Sound and Vibration, vol. 330, no. 1, pp. 87-109, 2011.

[4] R. Lotz and S. H. Crandall, "Prediction and measurement of the proportionality constant in statistical energy analysis of structures," The Journal of the Acoustical Society of America, vol. 54, no. 2, pp. 516-524, 1973.

[5] J. Woodhouse, "An introduction to statistical energy analysis of structural vibration," Applied Acoustics, vol. 14, no. 6, pp. 455$469,1981$.

[6] P. W. Smith, "Statistical models of coupled dynamical systems and the transition from weak to strong coupling," Journal of the Acoustical Society of America, vol. 65, no. 3, pp. 695-698, 1979.

[7] F. J. Fahy, "Statistical energy analysis: a critical overview," Philosophical Transactions of the Royal Society A: Mathematical, Physical \& Engineering Sciences, vol. 346, no. 1681, pp. 431-447, 1994.

[8] B. R. Mace, "Statistical energy analysis, energy distribution models and system modes," Journal of Sound and Vibration, vol. 264, no. 2, pp. 391-409, 2003.

[9] A. Le Bot and V. Cotoni, "Validity diagrams of statistical energy analysis," Journal of Sound and Vibration, vol. 329, no. 2, pp. 221$235,2010$.

[10] N. Lalor, "The experimental determination of vibrational energy balance in complex structures," in Proceedings of the Stress and Vibration: Recent Developments in Industrial Measurement and Analysis, vol. 1084 of Proceedings of SPIE, London, UK, July 1989.

[11] J. E. Manning, "Formulation of SEA parameters using mobility functions," Philosophical Transactions of the Royal Society A: Mathematical, Physical and Engineering Sciences, vol. 346, pp. 477-488, 1994.

[12] F. J. Fahy and P. P. James, "A study of the kinetic energy impulse response as an indicator of the strength of coupling between sea subsystems," Journal of Sound and Vibration, vol. 190, no. 3, pp. 363-386, 1996.

[13] P. P. James and F. J. Fahy, "Modal interaction indicator for qualifying Cs as an indicator of strength of coupling between sea subsystems," Journal of Sound and Vibration, vol. 235, no. 3, pp. 451-476, 2000

[14] R. J. M. Craik, "Non-resonant sound transmission through double walls using statistical energy analysis," Applied Acoustics, vol. 64, no. 3, pp. 325-341, 2003.

[15] R. S. Langley and V. Cotoni, "Response variance prediction in the statistical energy analysis of built-up systems," Journal of the Acoustical Society of America, vol. 115, no. 2, pp. 706-718, 2004.

[16] M. Robinson and C. Hopkins, "Transient statistical energy analysis: a two-subsystem model to assess the validity of using steady-state coupling loss factors for plate radiation," in Proceedings of the 18th International Congress on Sound and Vibration (ICSV '11), pp. 1517-1524, Rio de Janeiro, Brazil, July 2011. 
[17] R. Langley, "Recent advances and remaining challenges in the statistical energy analysis of dynamic systems," in Proceedings of the 7th European Conference on Structural Dynamics, Southampton, Southampton, UK, July 2008.

[18] B. R. Mace, "Power flow between two continuous onedimensional subsystems: a wave solution," Journal of Sound and Vibration, vol. 154, no. 2, pp. 289-319, 1992.

[19] K. Renji and M. Mahalakshmi, "High frequency vibration energy transfer in a system of three plates connected at discrete points using statistical energy analysis," Journal of Sound and Vibration, vol. 296, no. 3, pp. 539-553, 2006.

[20] F. J. Fahy and H. M. Ruivo, "Determination of statistical energy analysis loss factors by means of an input power modulation technique," Journal of Sound and Vibration, vol. 203, no. 5, pp. 763-779, 1997.

[21] C. Cacciolati and J. L. Guyader, "Measurement of SEA coupling loss factors using point mobilities," Philosophical Transactions of the Royal Society of London A: Mathematical, Physical and Engineering Sciences, vol. 346, pp. 465-475, 1994.

[22] S. De Rosa and F. Franco, "On the use of the asymptotic scaled modal analysis for time-harmonic structural analysis and for the prediction of coupling loss factors for similar systems," Mechanical Systems and Signal Processing, vol. 24, no. 2, pp. 455480, 2010.

[23] A. N. Thite and B. R. Mace, "Robust estimation of coupling loss factors from finite element analysis," Journal of Sound and Vibration, vol. 303, no. 3-5, pp. 814-831, 2007.

[24] L. Ji, B. R. Mace, and R. J. Pinnington, "A mode-based approach for the mid-frequency vibration analysis of coupled long- and short-wavelength structures," Journal of Sound and Vibration, vol. 289, no. 1-2, pp. 148-170, 2006.

[25] E. Balmes, "Use of generalized interface degrees of freedom in component mode synthesis," in Proceedings of the International Conference of Modal Analysis (IMAC '96), pp. 204-210, February 1996.

[26] P. J. Shorter and R. S. Langley, "On the reciprocity relationship between direct field radiation and diffuse reverberant loading," Journal of the Acoustical Society of America, vol. 117, no. 1, pp. 85-95, 2005.

[27] P. J. Shorter and R. S. Langley, "Vibro-acoustic analysis of complex systems," Journal of Sound and Vibration, vol. 288, no. 3, pp. 669-699, 2005.

[28] J. Cheng, X. Zhou, Y. Li, F. Sui, and L. Ji, "A fundamental investigation on extending statistical energy analysis modeling technique to the mid-frequency vibration regime," in Proceedings of the 18th International Congress on Sound and Vibration, Rio de Janeiro, Brazil, July 2011. 


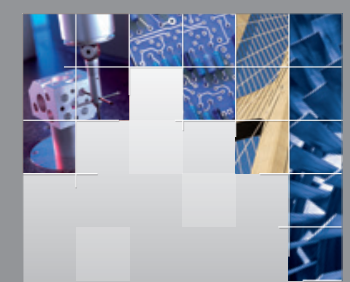

\section{Enfincering}
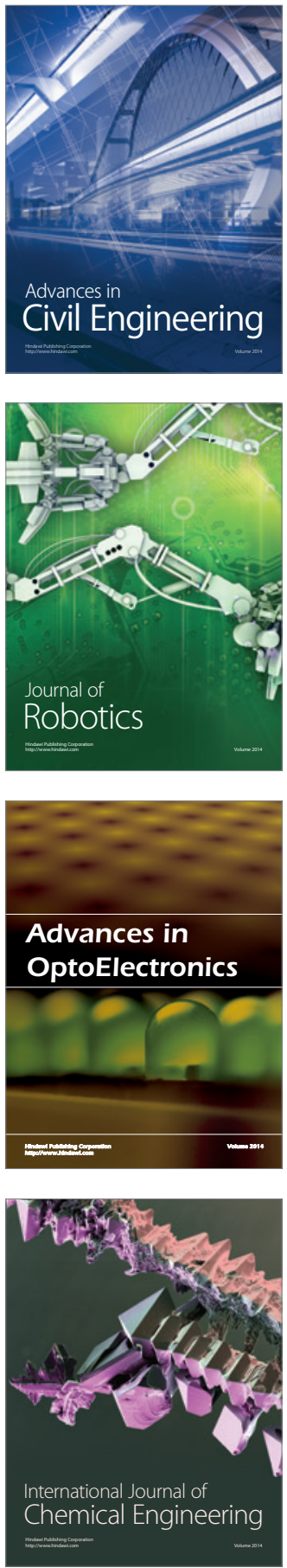

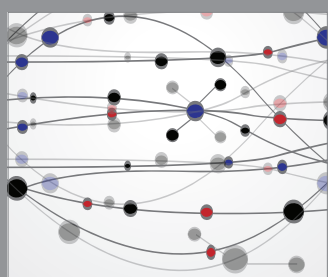

The Scientific World Journal

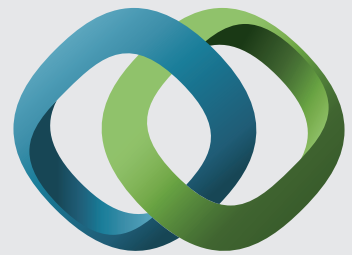

\section{Hindawi}

Submit your manuscripts at

http://www.hindawi.com
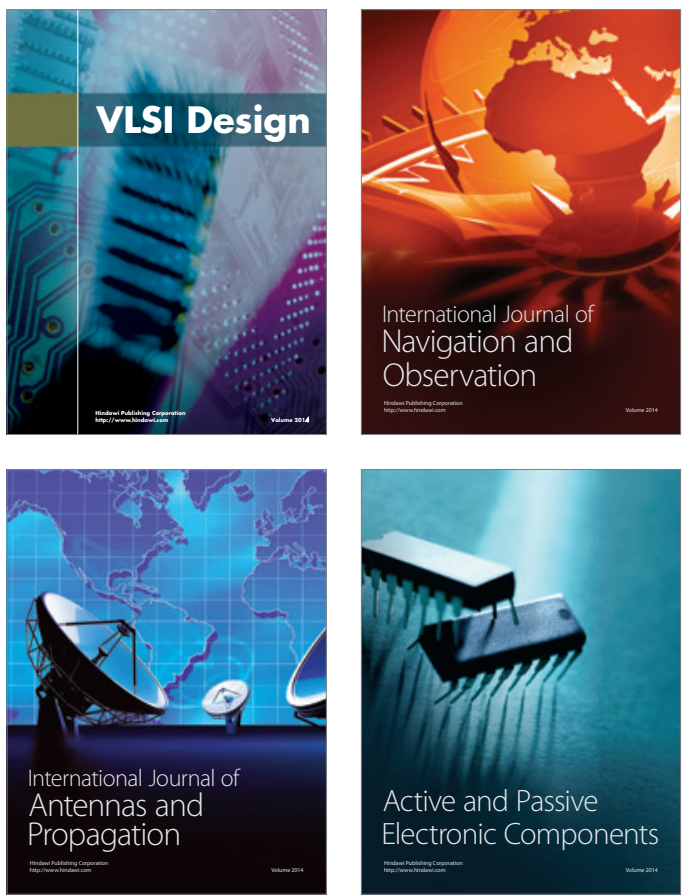
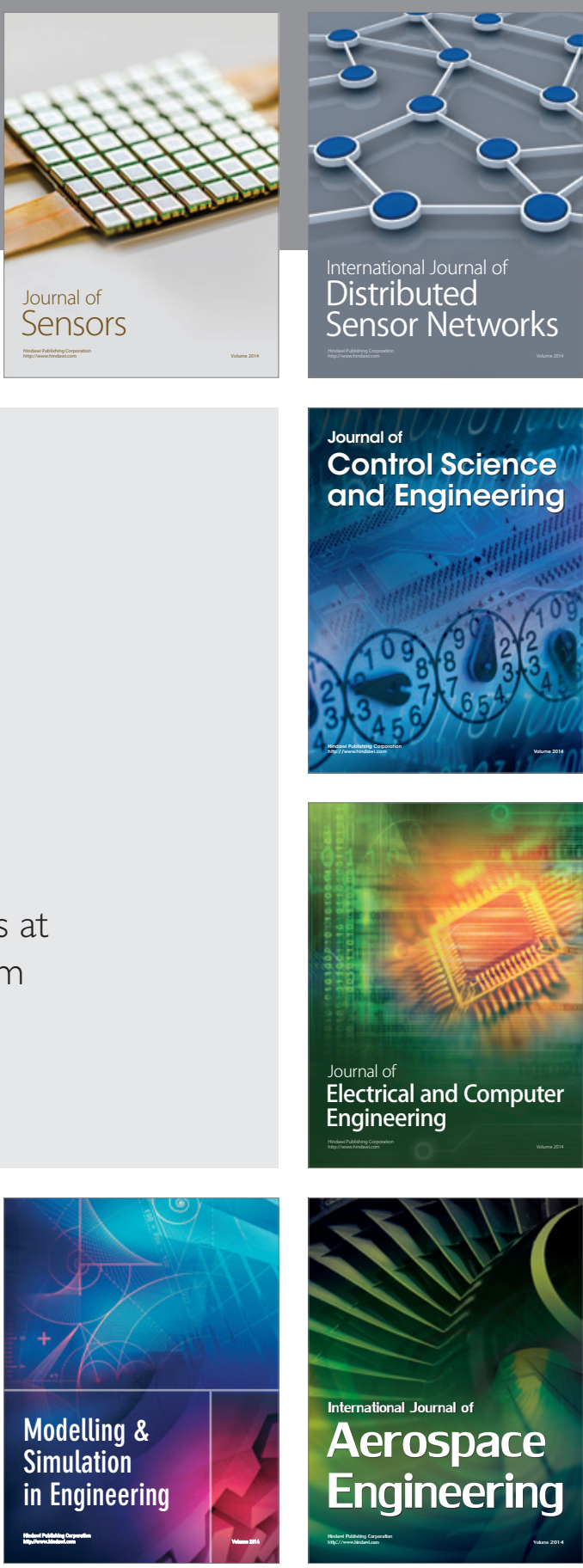

International Journal of

Distributed

Sensor Networks

Journal of

Control Science

and Engineering
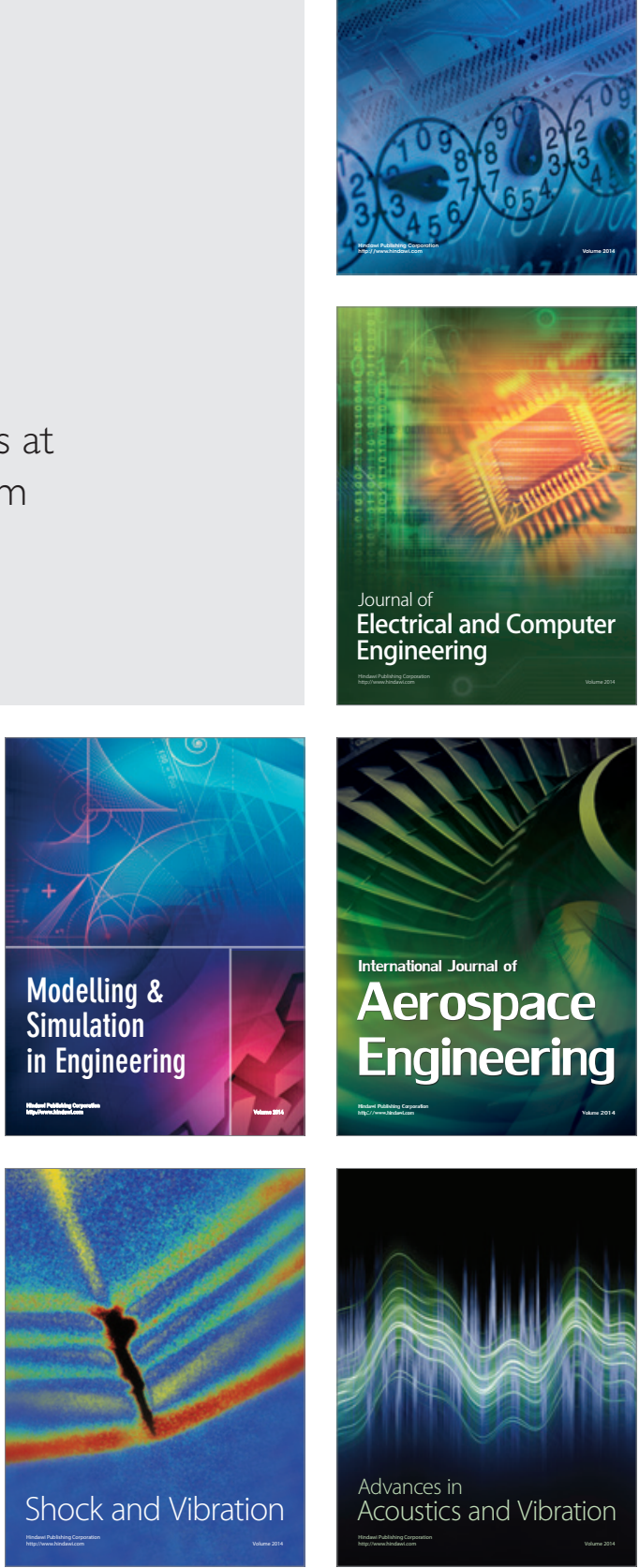